\title{
Incidence and risk factors for subcutaneous emphysema during robot-assisted laparoscopic prostatectomy
}

Chie Hanayama, M.D., Atsuyuki Hosono, M.D., Tsuyoshi Imaizumi, M.D., Shinju Obara, M.D., Tsuyoshi Isosu, M.D., Masahiro Murakawa, M.D.

Fukushima Medical University, Fukushima, Japan.

\section{Background :}

Subcutaneous emphysema (SE), is a complication of laparoscopic surgery that occasionally impairs ventilation and can cause a delay in postoperative recovery. In this study, we investigated the incidence and risk factors of SE in robotassisted laparoscopic prostatectomy (RALP).

\section{Materials and Methods:}

The subjects of the present study, which was approved by the ethics committee of our university (No. 2998), were 155 individuals patients who underwent RALP between January 2015 and December 2016.

The presence of SE was examined retrospectively in chest $X$ rays taken at the end of surgery. SE was classified into four grades based on, emphysema limited to under the thoracic diaphragm (Grade I), reaching the lower half of the thorax (Grade II), limited to the thorax (Grade III), and extended to the neck (Grade IV).
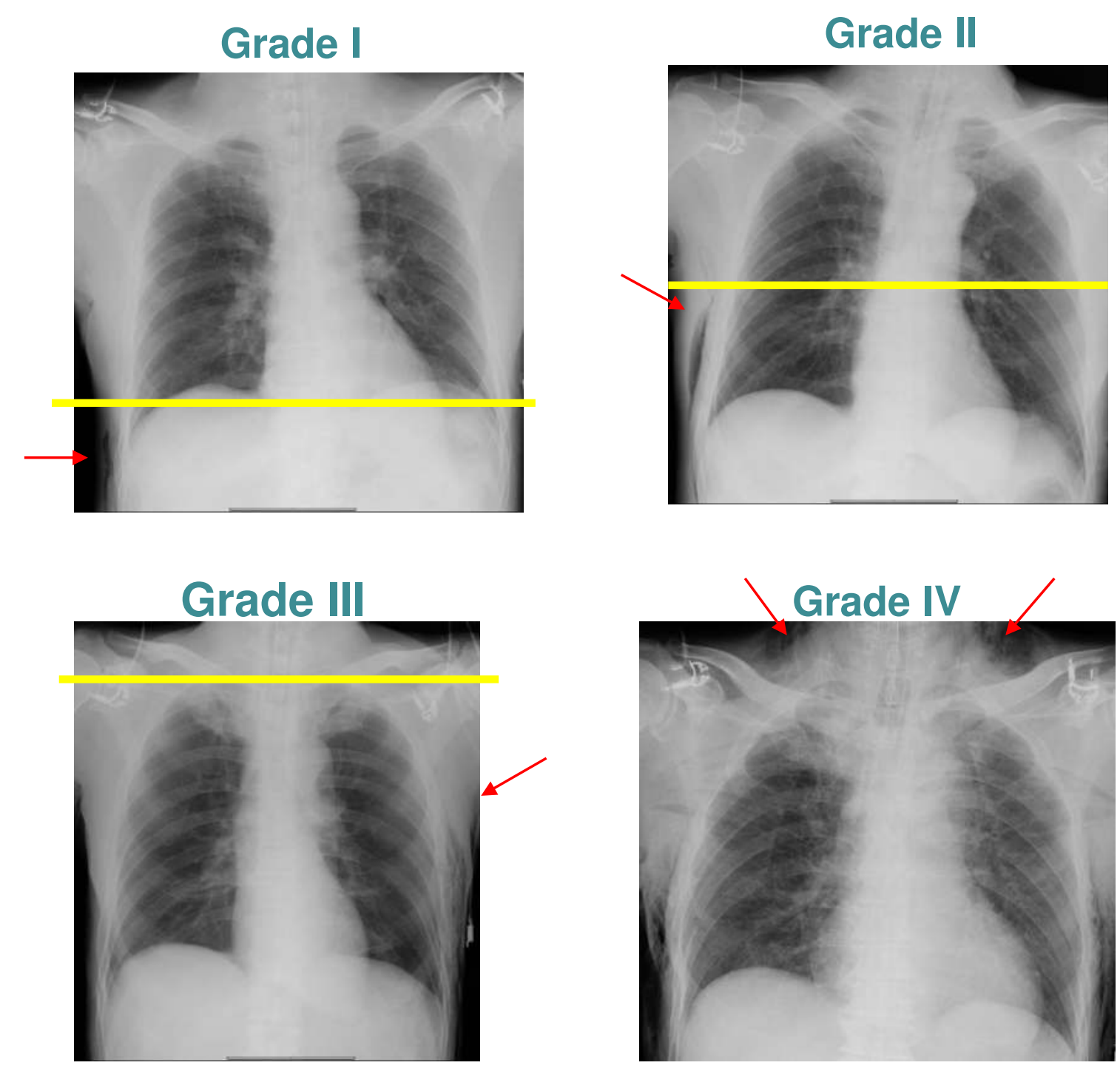

Age, height, body weight, BMI, ASA PS, preoperative albumin level, diabetes as a complication, and surgical duration were compared in patients who were and were not affected with SE, using a Mann--Whitney U test or chi-squared test. Data were analyzed by univariate analysis and then subjected to multivariate analysis using multiple logistic regression. $\mathrm{P}<0.05$ was considered to be significant in all analyses. The study was approved by the ethics committee of our university (No. 2998).

\section{Result :}

SE occurred in 47 of 155 cases (30.3\%) and was classified as Grades I, II, III and IV in 14 (9.0\%), 18 $(11.6 \%), 10(6.5 \%)$, and 5 (3.2\%) cases, respectively(table1).

The SE-affected group had a significantly lower height $(p=0.005)$, lower body weight $(p<0.001)$, and lower BMI $(p<0.001)$ compared to the unaffected group(table2)

\begin{tabular}{cccc}
\hline \multicolumn{4}{l}{ Table 2. Univariate analysis } \\
\hline Number & $\mathrm{SE}(-)$ & $\mathrm{SE}(+)$ & $P$-value \\
\hline Age & 109 & 14 & \\
Height $(\mathrm{cm})$ & $165.7(164.0-70.0)$ & $67(66.0-69.5)$ & 0.168 \\
Body weight $(\mathrm{kg})$ & $65.7(61.0-72.2)$ & $65.2(63.1-70.7)$ & $<0.001$ \\
BMI $\left(\mathrm{kg} \cdot \mathrm{m}^{-2}\right)$ & $23.9(22.3-26.1)$ & $25.0(22.9-25.7)$ & $<0.001$ \\
Albumin level $(\mathrm{g} / \mathrm{ml})$ & $4.3(4.2-4.5)$ & $4.3(4.0-4.5)$ & 0.258 \\
Diabetes & $27.52 \%$ & $19.10 \%$ & 0.547 \\
Surgical duration $(\mathrm{min})$ & $241(214-271)$ & $247(223-274)$ & 0.481 \\
\hline
\end{tabular}

Data are presented as median (range) or percentage

Multivariate analysis suggested that body weight is the factor significantly influenced the occurrence of SE(table3). And in comparison with each grade of SE, the average of the body weight is the more light in higher grade(Fig.1).

\begin{tabular}{ccccccc}
\hline \multicolumn{7}{l}{ Table 3. Logistic regression analysis } \\
\hline Factor & Coefficient & SE & Wald $\chi 2$ & P-value & OR & $95 \% \mathrm{Cl}$ \\
\hline Intercept & 10.419 & 6.180 & & & & \\
Height & -0.035 & 0.041 & 0.738 & 0.390 & 0.966 & $0.890-1.045$ \\
Body weight & -0.086 & 0.027 & 9.971 & 0.002 & 0.917 & $0.867-0.966$ \\
\hline
\end{tabular}

Fig.1 The relationship between SE grade and body weight

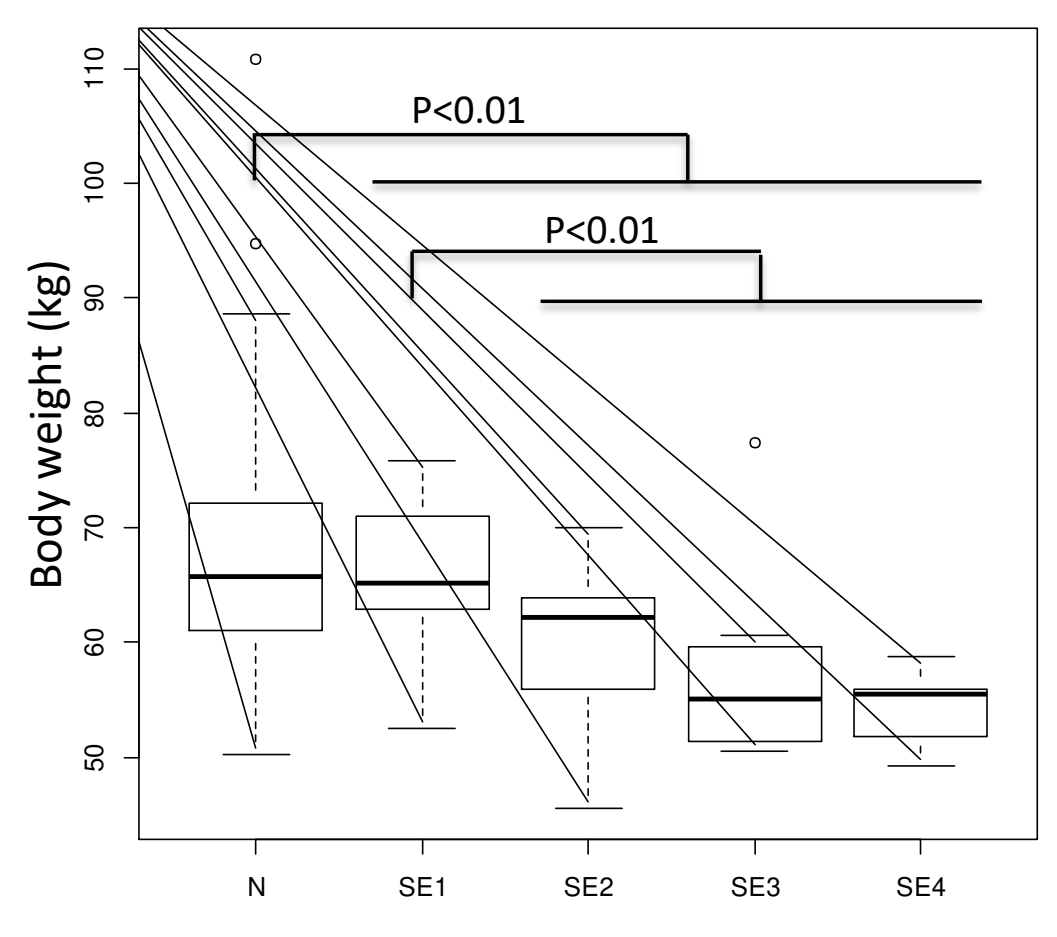

Discussion:

In this study,SE occurred in $30.3 \%$ of patients underwent RALP. This incidence of SE is higher than that in previous reports on laparoscopic surgery (0.3-3.9\%); however, past reports have included only marked cases of SE and it is likely that the incidence would have been closer to that in other laparoscopic surgeries if mild cases had also been included. Age, number of ports, and surgery duration have been proposed to affect SE, but these factors were not related to SE in our patients because the study included cases with a similar age and a single disease that were treated by one surgical team, with a consistent number of ports and little variance in surgery duration.

In univariate and multivariate analysis indicate that low body weight is the risk factor of SE at RALP. And the more light body weight is, the more seriously SE become.

\section{Conclusion:}

RALP may have a higher incidence of SE than that found in other laparoscopic surgeries. And it considered that SE occurrence is subject to low body weight.

\section{References:}

1) Ott Douglas E. Subcutaneous Emphysema: Beyond the Pneumoperitoneum.

JSLS(2014)18:1-7

2) Yoichi A., Shin E., Toshiro T., et al. Morbidity of laparoscopic radical prostatectomy: Summary of early multi-institutional experience in Japan. International Journal of Urology (2003) 10,430-4

3) Kosuke T., Takahisa G., Mariko A., et al. Scrotal Emphysema during Robotic Assisted Laparoscopic Prostatectomy: a case report. J Clin Anesth Jpn(2014) 38,1593-4 